Hilde Benjamin ergänzte, dass die Lage älterer Frauen, die ihren Lebensplan nachträglich nicht mehr ändern konnten, nicht durch eine formale und kompromisslose Umsetzung der Gleichheitssätze verschlechtert werden durfte. ${ }^{44}$ Den Ausschlag gab eine Befragung von 4000 Arbeitern zu der Frage, wie sie in der Ehe das Güterrecht praktizierten. Ganz überwiegend äußerten sich die Befragten dahin, dass sie den gemeinsamen Eigentumserwerb in der Ehe als selbstverständlich und gerecht ansahen. ${ }^{45}$ Und so führt das Familiengesetzbuch am 1. April 1966 die Errungenschaftsgemeinschaft als einzigen zwingenden Güterstand ein. ${ }^{46}$ Flankiert wurde diese güterrechtliche Regelung durch den im Zivilgesetzbuch verankerten Grundsatz, dass Eheleute nur gemeinsam Eigentum an Grundstücken erwerben können.

\section{Wiedervereinigung und stilles Begräbnis der Errungen- schaftsgemeinschaft}

Der Einigungsvertrag sah 1990 zunächst vor, dass die Eheleute aus dem Beitrittsgebiet den Güterstand der Errungenschaftsgemeinschaft beibehalten konnten, wenn ein Ehegatte bis zum 3. Oktober 1992 der Überleitung in den Güterstand der Zugewinngemeinschaft widersprach. Da für die rund acht Millionen Ehepartner ${ }^{47}$ kaum eine öffentliche Aufklärung über die Folgen etwaiger Untätigkeit stattfand, opferte man die Errungenschaftsgemeinschaft dem erklärten Ziel, schnell bundesweit einheitliche Güterrechtsverhältnisse herzustellen. ${ }^{48}$ Fast schon wütend beschrieb Martin Lipp die faktische und ersatzlose Abschaffung der Errungenschaftsgemeinschaft als vertane Chance. ${ }^{49}$ Die Beratungsliteratur war indes in einem Punkt einig: Welcher Güterstand für die Eheleute der günstigste sei, könne nicht allgemein, sondern nur anhand der konkreten Lebenssituation der Eheleute beurteilt werden. ${ }^{50}$

\section{Schluss}

Die Gesetzgebung zum Ehegüterrecht arbeitet seit jeher im Spannungsfeld zwischen gelebter Realität und gewünschtem Idealzustand der Ehe. In dem Bestreben, den gesetzlichen Güterstand so auszugestalten, dass er auf die meisten der eingegangenen Ehen passt, spielten häufig die Idealbilder der Ehe eine wichtigere Rolle als die Lebenswelten der Eheleute. Der Umgang der Gesetzgebung mit dem Ehegüterrecht unterliegt einem mit dem Ansteigen der Scheidungsquote geschuldeten
Wandel. Während der Fokus noch bis Mitte des 20. Jahrhunderts auf der Gestaltung der ehelichen Lebensverhältnisse, dem Gläubigerschutz und dem Erbrecht lag, erhalten Aspekte der Abwicklung der ehelichen Vermögensgemeinschaft nach Scheidung immer mehr Gewicht. Da die betroffenen Menschen sich während einer funktionierenden Ehe überraschend schlecht informiert über die automatischen Folgen einer Eheschließung zeigen und oft allein die Scheidung das Interesse an den güterrechtlichen Konsequenzen weckt, wird Ehegüterrecht zunehmend als Auflösungsrecht begriffen. Das zeigt sich auch an der seit 2009 in Kraft getretenen Reform, die vor allem die Beweisschwierigkeiten im gesetzlichen Güterstand der Zugewinnausgleich aufgegriffen hat. Es dürfte an der Zeit sein, den Blick wieder auf die funktionierende Ehe zu richten und den Eheleuten mit der Errungenschaftsgemeinschaft einen Güterstand zur Verfügung zu stellen, der eine gleichberechtigte Teilhabe während des Zusammenlebens sicherstellt. Denn zwei Drittel aller Ehen in Deutschland werden nicht durch Scheidung, sondern durch Tod beendet.

44 Grandke, Anita, Die Entwicklung des Familienrechts in der DDR, Berlin 2010, S. 28, 102 f., <http://edoc.hu-berlin.de/oa/reports/reeaYtqKfKxlQ/PDF/20eFhgZyFh7H2.pdf> (Zugriff: 30.9.2012).

45 Ebd., S. 114.

46 Brudermüller, Gerd/Wagenitz, Thomas, Das Ehe- und Ehegüterrecht in den neuen Bundesländern, FamRZ 1990, S. 1294-1300 (S. 1297).

47 Pawlowski, Hans-Martin/Lipp, Volker, Überlegungen zur Option für die Zugewinn- oder die Errungenschaftsgemeinschaft, FamRZ 1992, S. 377-382 (S. 377).

48 Bosch, Friedrich Wilhelm, Familien- und Erbrecht als Themen der Rechtsangleichung nach dem Beitritt der DDR zur Bundesrepublik Deutschland - verbunden mit einem Vergleich zweier deutscher Familien- und Erbrechtsordnungen, Güterrecht, FamRZ 1991, S. 1001-1011 (S. 1004).

49 Lipp, Martin, Die Eigentums- und Vermögensgemeinschaft des FGB und der Einigungsvertrag - eine vergebene Chance für eine Reform des Güterstandsrechts?, FamRZ 1996, S. 1117-1124 (S. 1119 f.).

50 Pawlowski, Hans-Martin/Lipp, Volker, Überlegungen zur Option für die Zugewinn- oder die Errungenschaftsgemeinschaft, a.a.O., S. 377-382; Bosch, Friedrich Wilhelm, Familien- und Erbrecht als Themen der Rechtsangleichung nach dem Beitritt der DDR zur Bundesrepublik Deutschland - verbunden mit einem Vergleich zweier deutscher Familien- und Erbrechtsordnungen, Güterrecht, FamRZ 1991, S. 1001-1011 (S. 1007-1009).

\title{
Die Errungenschaftsgemeinschaft in Deutschland - es gibt sie noch bzw. schon heute
}

\author{
Christiane A. Lang \\ Mitglied der Kommission Zivil-, Familien- und Erbrecht, Recht \\ anderer Lebensgemeinschaften des djb; Rechtsanwältin, Fach- \\ anwältin für Familienrecht, Berlin
}

Das Güterrecht scheint einer der Bereiche im deutschen Familienrecht zu sein, der stets nach Weiterentwicklung strebt. Wurde es doch erst vor drei Jahren in großen und wichtigen Teilbereichen reformiert, so nimmt die rechtspolitische Dis- 
kussion um den berechtigten Fortbestand der Zugewinngemeinschaft heute derart zu, dass man zeitweise fast meinen könnte, die nächste Reform sei schon zum Greifen nahe. Ungeachtet möglicher zukünftiger Reformbestrebungen aber gibt es sie schon bzw. noch heute, auch hierzulande: Die Errungenschaftsgemeinschaft in Deutschland und zwar nach den $\mathbb{\int} \int 39$ und $40 \mathrm{FGB} / \mathrm{DDR}$.

Das Familiengesetzbuch der DDR (FGB/DDR) kannte die Errungenschaftsgemeinschaft und sah die sogenannte Eigentums- und Vermögensgemeinschaft der Ehegatten vor. Mit der deutschen Wiedervereinigung und dem Inkrafttreten des BGB im Beitrittsgebiet zum 3. Oktober 1990 ging der Güterstand des FGB/DDR grundsätzlich von Gesetzes wegen in den gesetzlichen Güterstand der Zugewinngemeinschaft über. Anderes galt nur, wenn die Ehegatten einer zu DDR-Zeiten geschlossenen Ehe von ihrem Recht aus Artikel 234 \$ 4 Absatz 2 Satz 1 EGBGB Gebrauch machten und sich für die Fortgeltung des bisherigen DDR-Güterstands entschieden. ${ }^{1}$ In diesen Fällen wurde der bisherige DDR-Güterstand auch nach der Wiedervereinigung beibehalten. ${ }^{2}$ Aber auch für die „Güterstandswechsel-Ehen“ folgt aus dem Inkrafttreten des BGB nicht die Bedeutungslosigkeit des FGB/DDR. Denn die durch die Wiedervereinigung bedingte Änderung des gesetzlichen Güterstands trat erst mit Wirkung ex nunc ein und so sind für die eheliche Auseinandersetzung des bis zur Wiedervereinigung erworbenen Vermögens die Vorschriften nach dem FGB/DDR weiter anzuwenden. ${ }^{3}$ Im Scheidungsfall einer zu Zeiten der DDR geschlossenen Ehe ist mithin immer das zu DDR-Zeiten erworbene Vermögen nach den Regeln der Errungenschaftsgemeinschaft und somit nach den $\mathbb{S} \mathbb{S} 39$ und 40 FGB/DDR auseinanderzusetzen. ${ }^{4}$

Zunächst sind die Eigentumsverhältnisse der Eheleute am jeweils auseinanderzusetzenden Vermögen zu klären. Gemäß \ 13 Absatz 1 Satz 1 FGB/DDR gehören die von einem oder beiden Ehegatten während der Ehe durch Arbeit oder aus Arbeitseinkünften erworbenen Sachen, Vermögensrechte und Ersparnisse beiden Ehegatten gemeinsam. Das gemeinschaftliche Eigentum entsteht während der Ehe also kraft Gesetz und ist eine Form des Gesamteigentums ( $\mathbb{S} 34$ Abs. 2 i.V.m. $\int 42$ Abs. 3 ZGB). ${ }^{5}$ Das vor der Eheschließung allein von einem Ehegatten Erworbene, ihm Geschenkte oder durch Erbschaft Zugeflossene verbleibt indes in seinem Alleineigentum.

Sodann ist bei der Vermögensauseinandersetzung entsprechend der Vermögensverhältnisse zu unterscheiden: Ist das während der Ehe errungene Gemeinschaftseigentum auseinanderzusetzen, sieht $\mathbb{} 39 \mathrm{FGB} / \mathrm{DDR}$ eine Vermögensaufteilung zu gleichen Teilen in Natur vor. Die einzelnen Vermögensgegenstände sind dabei zu bewerten und möglichst genau aufzuteilen ${ }^{6}$ und einem Ehegatten zu Alleineigentum zuzuweisen ${ }^{7}$. Ist eine solche Teilung in Natur nicht möglich, wird an dem unteilbaren Vermögensgegenstand hälftiges Miteigentum begründet. In jedem Falle aber kommt dem sogennanten Halbteilungsgrundsatz wesentliche Bedeutung zu, sodass - sollte im Ausnahmefall eine ungleiche Verteilung entstehen - zusätzlich ein auf Geld gerichteter Erstattungsanspruch desjenigen begründet wird, der den Vermögensgegenstand zum geringeren Anteil zugeteilt erhält ( $\mathbb{S} 39$ Abs. 1 S. 3 FGB/DDR). ${ }^{8}$

Ist bei Beendigung einer Ehe Alleineigentum auseinanderzusetzen, regelt $\mathbb{S} 40 \mathrm{FGB} / \mathrm{DDR}$ die Teilhabe des einen Ehegatten am Alleineigentum des anderen. Im Gegensatz zu $\mathbb{} 39$ FGB/DDR ist keine Aufteilung des Vermögens in Natur vorgesehen. Vielmehr ist der Ausgleichsanspruch des $\mathbb{\$} 40$ FGB/ DDR von vornherein als ein Anspruch auf Geld konzipiert und dient dem Ausgleich für während der Ehe beigetragene Leistungen zur wesentlichen Mehrung oder Erhaltung des Alleineigentums des einen durch den anderen Ehegatten. ${ }^{9}$

Das FGB/DDR sieht also entweder eine Halbteilung des gemeinschaftlichen Vermögens oder eine Kompensation für eine mindestens erbrachte Werterhaltung des Alleineigentums unter den Eheleuten vor. Damit wurde eine güterrechtliche Auseinandersetzung im Sinne der Errungenschaftsgemeinschaft geschaffen, die heute trotz des (noch) „errungenschaftslosen" BGB Eingang in die Familienrechtspraxis der Bundesrepublik Deutschland gefunden hat und zur Anwendung gelangt.

1 Götsche, Frank, Die Anwendung der $\S \S 39$, 40 FGB/DDR bei der vermögensrechtlichen Auseinandersetzung im Scheidungsfall, FamRB 6/2003, S. 189, 190; Schael, Wolfgang, Zugewinnausgleich - Spezielle Rechtsprobleme in den neuen Bundesländern, NJ 7/2004, S. 289.

2 BGH v. 5.5.1999 - XII ZR 184/97, FamRZ 1999, 1197, 1198 = NJW 1999, 2520 ff.; Die Bedeutung für die familienrechtliche Praxis erlangte jedoch geringen Wert, da nur etwa 3.700 Ehepaare von ihrem Optionsrecht Gebrauch machten, vgl. Peters, Registerverfahrensbeschleunigungsgesetz, FamRZ 1994, 673, 674.

3 BGH v. 5.6.2002 - XII ZR 194/o0, FamRZ 2002, $1097=$ MDR 2002, $1068 \mathrm{f}$.

4 BGH v. 5.6.2002 - XII ZR 194/o0, FamRZ 2002, $1097=$ MDR 2002, 1068 f., in Fortführung von BGH v. 5.5.1999 - XII ZR 184/97, FamRZ $1999,1197,1198$ = NJW 1999, $2520 \mathrm{ff}$.

5 FGB-Kommentar, Staatsverlag der DDR, 5. Aufl. 1982, §13 Anm. 1.1 und 1.3.

6 BGH v. 29.1.1992 - XII ZR 241/90, FamRZ 1992, 531, 532 f.

7 FGB-Kommentar, Staatsverlag der DDR, 5. Aufl. 1982, § 39 Anm. 1.5 und 1.6.5.

8 BGH v. 15.1.1992 - XII ZR 202/90, FamRZ 1992, 414, 415 = NJW 1992, 821.

9 S. auch BGH v. 5.5.1999 - XII ZR 184/97, FamRZ 1999, 1197, 1198 = NJW 1999, 2520 ff.; vgl. § 40 Abs. 1, 2 FGB/DDR. 